\title{
Is Fear of Movement (FoM) in sports related activities a latent trait?: the item response model applied to the Photographic Series of Sports Activities for Anterior Cruciate Ligament Rupture (PHOSA-ACLR).
}

Wim van Lankveld ( $\nabla$ wim.vanlankveld@han.nl)

Hogeschool van Arnhem en Nijmegen Faculteit Gezondheid Gedrag en Maatschappij https://orcid.org/0000-0002-6102-2997

\section{Ron J. Pat-El}

The Open University of the Netherlandsion and Curriculum Partnerships

\section{Nicky van Melick}

Knee Expert Centre Eindhoven

\section{Robert van Cingel}

Sport Medisch Centrum Papendal

\section{J. Bart Staal}

Hogeschool van Arnhem en Nijmegen

\section{Research article}

Keywords: anterior cruciate ligament reconstruction (ACLR), Kinesiophobia, fear of harm/movement/injury, validation

Posted Date: December 3rd, 2019

DOl: https://doi.org/10.21203/rs.2.18117/v1

License: (c) (1) This work is licensed under a Creative Commons Attribution 4.0 International License. Read Full License 


\section{Abstract}

Background: Fear of Movement is an important factor predicting return to sports in Anterior Cruciate Ligament Reconstruction (ACLR). Fear for specific ACLR related movements can be addressed measuring the Photographic Sports Activities for ACLR (PHOSA-ACLR). This study was conducted to determine whether Fear of Movement assessed using the PHOSA-ACLR is a latent trait, and to analyse differences in PHOSA-ACLR in athletes with or without an ACL rupture.

Methods: Data from three groups of participants were included in this study: 1) ACLR patients participated in a previous study measuring psychological dimensions after $\operatorname{ACLR}(n=58) ; 2)$ first year physical therapy students at the HAN University of Applied Sciences $(n=169)$, and 3 ) junior football players that were enrolled in a training programme of the Professional Soccer club Vitesse (Arnhem) ( $n=$ 30). All participants completed the PHOSA-ACLR (scale score 0-10), and questions related to demographics. ACLR patients completed additional questionnaires to assess functioning and Fear of Harm. PHOSA-ACLR items were analysed with Item Response Theory using the Graded Response Model (GRM). Differences between three groups of participants were analysed using Univariate Analysis of Variance.

Results: The two-parameter GRM with both location and discrimination $(-2 \mathrm{LL}=-5485.05)$ showed a significantly better fit than the one-parameter model $(-2 L L=-5515.36),(1)=30.31, p=0.00)$. The items of the PHOSA-ACLR thus constitute a latent trait. The three groups of participants differed in reported fear of movement assessed with the PHOSA-ACLR after controlling for covariates gender and age. Physical therapy students reported higher levels of fear of movement compared to either ACLR patients and healthy soccer players (respectively $4.9(95 \% \mathrm{Cl} 4.6-5.3) ; 3.6(95 \% \mathrm{Cl} 2.5-3.7) ; 2.6(95 \% \mathrm{Cl} 1.6-3.6))$.

Conclusion: PHOSA-ACLR items constitute a latent trait of fear of movement for ACLR specific movements. Contrary to expectations, PHOSA-ACLR is higher in first year physiotherapy students compared to patients rehabilitating from ACLR, and healthy junior soccer players. The PHOSA-ACLR might be useful for clinicians to identify high levels of fear of movement related to ACLR, and provide a tool to help the patient overcome fear of movement using exposure techniques.

\section{Background}

An anterior cruciate ligament (ACL) tear is a frequently reported injury in sports activities that include pivoting movements of the knees, such as soccer, basketball, football, handball, and skiing. The incidence of ACL tears is estimated at 68.6 per 100,000 person years[1]. Most patients recover to normal function within 6-8 weeks [2], but only half of the patients return to pre-injury sports levels [3, 4]. Fear of Movement (FoM) or (re)injury is an important factor in rehabilitation after sports related injury $[5,6]$. There are several pathways by wich FoM can have an impact on recovery after a sports related injury like ACLR[5]. Physiological arousal associated with fear can induce increased muscle tension, fatigue, decreased coordination, and muscular guarding thus escalating the individual's susceptibility to injury[7]. 
Trigsted et al.[8] showed that fear of re-injury is associated with stiffened movement patterns associated with increased risk of a second ACL injury. The fear avoidance model of pain offers another pathway in which FoM can hinder recovery[9]. The model holds that averse events might trigger negative emotions and cognitions that can become conditioned. In this way FoM can become internalized preventing that person's engagement in activities necessary for recovery[9]. In ACRL rehabilitation, FoM has a negative impact on return to sports after ACL rupture and reconstruction[10,11]. However, intensity of these fears differs between individuals from non-existent to maladaptive psychological outcomes and disorders (anxiety disorders), as in kinesiophobia $[12,13]$. Therefore, it is important to assess the individual patient's level of FoM after ACLR.

In those ACLR patients with disruptive levels of FoM, addressing task specific FoM in rehabilitation might help patients return to their preinjury sport or recreational activity[14, 15]. Fear can be treated using exposure, which has been successfully applied in chronic low back pain, complex regional pain syndrome and other conditions[16]. Graded exposure guides the patient through a hierarchical series of fear eliciting movements and activities that have been avoided. McArdle described a cognitive-behavioural approach based on exposure to treat dysfunctional FoM in one person with ACLR [17]. However, results of exposure treatment has not been reported in the ACLR rehabilitation literature. One reason might be the lack of instruments to determine a hierarchy of perceived harmfulness of physical activities and movements specific for ACLR. Fear in ACLR is most frequently assessed using generic measures of Fear of Movement, such as the Tampa Scale of Kinesiophobia (TSK)[18], or the ACL-Return to Sports Injury Scale (ACL-RSI)[19]. However, both scales do not provide information about specific FoM induced by specific sport related tasks in the individual with ACLR, preventing return to sports. Therefore, the Photographic Sports Activity-Anterior Cruciate Ligament Reconstruction (PHOSA-ACLR) was developed to assess fear for specific sports related activities[20]. Although strongly associated with the FoM assessed with the TSK, the PHOSA-ACLR gives specific information about fear invoking sports situations that is not measured by other measures. Traditional psychometric properties such as construct validity, internal consistency and test-retest reliability underlined the PHOSA-ACLR's validity and reliability[20]. However, an important limitation of these traditional psychometrics is the assumption that measurement precision is constant across the entire trait range, or that each item in a test contributes equally to the test score[21]. As a result measurement precision may not be constant for all respondents, as is often true in practice. Especially tests used for clinical purposes are good at differentiating among people in the high range of a trait, but less well-suited for differentiating people in the normal-to-low range of the trait, which is where the at-risk patients usually reside at. For clinical use it is important to know how the individual items on the PHOSA-ACLR are related to each other hierarchically based on respondents' responses. Based on such a fear hierarchy of individual items, clinicians can use the instrument in exposure treatment for these patients. To establish such a hierarchy of items latent trait analysis using Item Response Theory (IRT) can be used. IRT allows for the identification of items that are indicative of high ability, and potentially how well these items discriminate around that ability. The better the item discriminates the stronger its diagnostic information and thus value the item has in estimating FoM in clinical practice. 
Therefore, this study was conducted to determine whether FoM assessed using the PHOSA-ACLR is a latent trait using Item Response Theory Models. Furthermore, differences in PHOSA-ACLR score between patients with a recent ACLR and healthy athletes was determined. It is expected that patients with ACLR will have higher scores on FoM assessed with the PHOSA-ACLR compared to healthy controls.

\section{Methods}

\section{Participants.}

For this study we used data from three samples of subjects. The first sample consisted of 58 ACLR patients, who participated in a study conducted in 2015 to measure psychometric dimensions relevant to FoM after ACLR[20]. Patients were included from physical therapy practices when they were between 3 months and 3 years after their ACLR. The second sample of respondents were 169 healthy regular students at the HAN University of Applied Sciences. All first year physical therapy students attending a lecture were asked to complete the ACLR-PHOSA. At the start of the lecture, students were informed about the study, and asked to provide written informed consent. The third group of participants were 30 healthy junior soccer players that were enrolled in a training programme of the professional Soccer club Vitesse (Arnhem), additional to their regular educational programme.

\section{Measurements.}

All participants reported demographics (gender, age), and completed the PHOSA-ACLR using an online questionnaire (sample 1) or printed questionnaire (sample 2 and 3). The PHOSA-ACLR is comprised of photographic images of 12 sports situations invoking FoM in ACLR patients. In the introduction to the questionnaire participants are asked to imagine performing the movement depicted in the photograph and then they are asked to report the subjective FoM for each item on a scale from 0-10 (11 point rating scale). A score of 0 means 'totally not damaging', and 10 stands for 'very damaging'. The average item score for the scale is computed. In a previous study the PHOSA-ACLR showed excellent reliability and validity in patients with ACLR[20].

Sample 1 (ACLR patients) was asked to report time since reconstruction and completed two additional questionnaires assessing self-reported functioning and fear of movement. Self-reported functioning was assessed using the validated Dutch version of the Knee Injury and Osteoarthritis Outcome Score (KOOS) [22]. The KOOS measures pain, other disease-specific symptoms, activities of daily life (ADL) function, sport and recreation function, and knee-related quality of life (QOL). For each scale the scores were recoded from $0-100$, with 100 depicting no problems. Fear of movement was assessed using the Dutch version of the Tampa Scale of Kinesiophobia (TSK) $[18,23,24]$. The TSK measures generic fear of movement or (re)injury using 17 items. Item scores range from $1-4$ where $1=$ strongly disagree and $4=$ strongly agree. Total score is the count of all 17 items after inversion of items 4, 8, 12 and 16. A score higher than 36 is considered kinesiophobia. 
Healthy subjects in sample 2 and 3 were asked to complete two additional questions related to sports activities (which sports activities they were active in, and hours of sports per week), and three yes/no questions relate to physical condition (do you have a current medical condition, have you ever had previous lower extremity injuries, and do you know anyone with an ACL rupture).

\section{Analysis.}

Descriptive statistics are given for the participants in the three groups. For continuous variables mean score is reported, standard deviation, and 95\% Confidence Interval for Means (95\% Cl) when appropriate. For dichotomous variables percentages are given for each group. For sample 1 ACLR related mean scores are given for functioning (KOOS), and Fear of Movement (TSK). In sample 2 and 3, reported sports activities were recoded into two categories: low risk ACL tear sports (code 1 = swimming, horseback riding, ballet, running, etc.); and high-risk ACL tear sports (code 2 = soccer, hockey, tennis, baseball, etc.).

Item Response Theory (IRT) models are used to determine the probability of an individual's response to an item, given their latent ability, which in this case would be ACLR specific FoM [24]. In this study we applied a polytomous IRT-model called a Graded Response Model (GRM) to the PHOSA-ACLR in which the item parameters were estimated for each 11-point Guttman item used in this study. The GRM is an especially useful item response model when item response options can be conceptualized as ordered categories with a strongly restricted monotonicity, such as Guttman scales. Item locations are the polytomous equivalent to item difficulties in IRT models for dichotomous responses. A GRM is similar to IRT models with dichotomous outcomes, but within the GRM framework an item response scale reconstructs a polytomous scale in a series of $m-1$ dummy response dichotomies, where m represents the number of response options for a given item. Thus, an item rated on a 11-point scale has ten response dichotomies: category 1 versus category 2, category 2 versus category 3 , etc. The graded response model estimates the probability of endorsing a response category for each item conditional on the latent trait, in which the latent trait is considered a weighted and standardized total scale score. Two models were tested: The first model was a 1-parameter (1-PL) model, in which only the item locations were estimated, and discrimination was set to one for all items (often referred to as a Rasch-model). The second model that was tested was a 2-parameter model in which in addition to the estimation of item locations the item discrimination-parameters were estimated. Item discrimination equates to the slope of the item response function, and is a measure of how sharply the response options discriminate between each other around their location parameters. Higher discrimination means that the items are more informative. To determine whether the added complexity of the 2PL model is necessary the fit of the two models were compared on their -2loglikelihood, which is $\chi^{2}$ distributed. By testing whether the chi-square is significant it was determined if the added complexity of the 2PL model significantly improved the model fit.

Differences between groups in PHOSA-ACLR. Differences between groups were tested with Univariate Analysis of Covariance (ANCOVA). Before running an ANCOVA possible covariates have to be identified. Covariates are variables that differ between groups under study and that are related to the dependent 
variables. Differences between samples in demographics were tested using $\chi^{2}$ for dichotomous variables, and ANOVA for continuous variables. Associations between demographic variables and PHOSA-ACLR scores were calculated using $\chi^{2}$ for dichotomous variables and Pearson correlation for continuous variables. Correlations were interpreted as small $(r=.10)$, medium $(r=.30)$, and large $(r>.50)$ [25]. Normality of items distribution were tested using the Shapiro-Wilks test. Levene's test of equality of error variance was used to test the hypothesis that the error variance of the dependent variable (PHOSA-ACLR) is equal across groups. $F$ statistics and significance level are given for the corrected model comparing the three groups of participants controlling for covariates (ANCOVA). The same statistics are given for the independent variable (groups to be compared), and covariates. For significant co-variates partial eta square was computed to determine the percentage of variance attributed to that covariate. All analyses were done using SPSS version 26 (IBM Corporation). A p-value $<0.05$ was used as an indication of statistical significance.

\section{Results}

\section{Description of the samples.}

Sample 1 included 58 ACLR patients (mean age 25.9 years; range 17-56; SD = 8.2; 43\% male). Average time since reconstruction was 15.5 months (range $3-36, S D=8.0$ ). In Table 1 mean levels of selfreported functioning (KOOS), and fear of movement (TSK) are given.

Table 1 shows that patients in the ACLR group on average scored highest on the KOOS scales Pain and $A D L$ as compared to the other KOOS scales, indicating little pain, and high levels of ADL functioning. KOOS QOL showed relative lower scores indicating that quality of life was limited despite good recovery of pain and functioning. Based on the TSK score, kinesiophobia (TSK score $>36$ points)was present in 26 patients, or $45 \%$ of the patient sample.

Table 2 shows descriptives of both samples of healthy participants. Sports activities reported by students were recoded into high risk and low risk sports activities.

Sample 2 included 169 regular students (mean age $=19.2, \mathrm{SD}=2.0 ; 48 \%$ male). Students participated frequently in sports activities. Only 6 students (3.4\%) reported that they did not actively participate in sports activities. On average PT students reported to be active in sports during 5.6 hours a week. Sample 3 comprised of 30 soccer players from Vitesse (mean age $=18.3$, range $-17-20 ; S D=3.2 ; 94 \%$ males). In this group of soccer players only two persons were female. This group reported an average of 14.9 hours of active sporting per week. The three groups of participants differed in average age $(F(2,254)=51.2, p<$ 0.001 ), with ACLR patients being of higher age compared to the student groups. The three groups of participants differed in gender $\left(\chi^{2}=21.4, p<.001\right)$. The group of junior soccer players were predominantly male, with only two females. Group 2 differed in percentage high/low risk sports compared to both other groups, where all participants in group 1 and 3 were considered high rick athletes. 
Prior to analysis, the PHOSA items were examined for accuracy of data entry, and missing values. No unior multivariate outliers were found. The dimensionality of the PHOSA was tested with Principal Axis Factoring, which showed a clear unidimensional fit for the PHOSA items (TLI = 0.90, RMSR =0.05) with factor loadings between 0.69 and 0.89. Descriptive statistics for the PHOSA items are summarized in Table 3.

The graded response model was used to calibrate item parameter estimates for the PHOSA given its Guttman-scaling; i.e. ordered polytomous response format. The best fitting GRM was first assessed by comparing two models, (1) a one-parameter model (Rasch) with location as parameter (and discrimination assumed fixed to 1); and (2) a two-parameter model, with both location and discrimination as parameters. The two-parameter model $(-2 \mathrm{LL}=-5485.05)$ showed a significantly better fit than the one-parameter model $(-2 L L=-5515.36), \chi 2(1)=30.31, p=0.00)$. Subsequent analyses pertain to the fit of the two-parameter model in which both location and discrimination are estimated. In Table 4 the item difficulty locations are sorted, from top to bottom, in ascending order of increasing difficulty.

How these locations relate to the thresholds within each item are summarized in Item Information Curves in Figure 1.

The ICCs in figure 1 indicate that items 8 and 10 are most informative in the range of -2 and 2, whereas item 12 is most informative below -2 , and item 9 above +3 . Item 6 is least informative in general. The thresholds in Table 2 correspond to the intersection between curves. At these intersection it is equally likely a person will be classified into adjacent categories, and therefore to obtain one of two successive scores on the item. The mean of the threshold locations within an item is represented by the location parameter. The locations range from 1.69 to 3.48 logits, where "Hopscotch" (PHOSA_8) was the most difficult item and "Sliding" (PHOSA_6) was the easiest item (table 4).

In Figure 2 the distributions of ability estimates in the sample are summarized in a kernel density plot showing an approximately normal distribution slightly negatively skewed.

The kernel density plot shows that relatively high PHOSA-scores are rare, whereas most of the respondents are either slightly above average or well below average. The Test Item Information Function, visualized in Figure 3 shows that the test is most informative (has its peak-information) for people within a standardized PHOSA-range of -2 to +2 standard deviations, with an evenly distributed peak in that range, indicating that the test is consistently reliable in this entire range.

\section{Difference between groups in FoM assessed with the PHOSA-ACLR.}

Levene's test of variance was significant $(F(2,253)=5.1) ; p=0.007)$ indicating that the error variance is not equal across groups. The one sample Shapiro Wilks was significant indicating that the distribution of PHOSA-ACLR scores was not normal. Table 6 depicts average PHOSA-ACLR scores for patients of each of the three samples, without any correction of covariates. 
On average, regular students reported the highest level of FoM assessed using the PHOSA-ACLR comparable with FoM reported in soccer players. Regular students on average reported higher levels of FoM compared to ACLR patients and soccer players. Within the healthy samples (samples 2 and 3 ) students participating in high risk sports did not differ from students with low risk sports activities in average PHOSA-ACLR scores; mean PHOSA-ACLR scores in the high risk group is 2.06 (SD 0.22), compared to $2.4(S D=0.23)$ in the low risk group $(F(2,190)=4.47, p=n s)$. A chronic health condition was reported by 17 students, and these students did not report different PHOSA-ACLR scores when compared to students without chronic condition. Over one third of the students ( $N=73 ; 38 \%)$ reported to know someone with an ACL rupture. These students did not differ in mean PHOSA-ACLR from students who did not know anyone with an ACL rupture. Number of hours spent on sports on average per week showed weak association with PHOSA-ACLR $(\mathrm{N}=198 ; r=-.30, p<.001)$. Students spending more time on sports showed on average lower scores on PHOSA-ACLR.

For all three samples gender, age and previous injury are known and analyzed as possible covariates. Average PHOSA-ACLR score for males is $3.8(\mathrm{SD}=2.3)$ compared to $4.7(\mathrm{SD}=2.2)$ in females $(\mathrm{t}(254)=$ $2.98, p<.0001)$. Age of participants showed a small but significantly reversed correlation with PHOSA$\operatorname{ACLR}(r=-0.21, p<.001)$. Previous injury at the knee was reported by 194 participants (including all participants from sample 1). Mean PHOSA-ACLR for these participants was $4.32(S D=2.3)$ and was not significantly different from the average score of $4.0(S D=2.6)$ in those participants not reporting previous injury $(t(254)=0.97, p=0.35)$. Therefore, only gender and age are considered as covariates in the ANCOVA for differences between the three groups, as these are related to PHOSA-ACLR, and differ between groups. In the ANCOVA analysis the covariate was unrelated to PHOSA-ACLR, with gender being the sole covariate with a significant relation to PHOSA-ACLR $(F(1,255)=4.01 ; p=.04)$. Gender explained $1.6 \%$ of the variance in PHOSA-ACLR independently from the other variables in the equation (partial eta squared $=0.016$ ). There was a significant difference between the three groups in PHOSA-ACLR after controlling for age and gender $(F(2,255)=17.1, p<.001)$. Differences in groups explained $12 \%$ of variation in PHOSA-ACLR (Partial Eta Squared $=0.12$ ). Post-hoc pairwise comparison adjusted for multiple comparisons (Sidak) showed that the students differed from both the ACLR group (lower FoM, mean difference $=1.6, p<.001$ ), and from soccer players (higher FoM, mean difference $=2.0, p<.001$ ). As the distribution of scores on the PHOSA-ACLR was shown to be not normal, the ANCOVA was repeated using normal transformation of PHOSA-ACLR, with similar results (data not shown).

\section{Discussion}

This study showed that the distribution of scores on the individual items of the PHOSA-ACLR fits the Item Response Model. It is therefore safe to assume that the individual items of the PHOSA-ACLR measure one underlying latent trait. The model showed good fit with "Sliding" (PHOSA_6) least likely to induce FoM, and "Hopscotch" (PHOSA_8) most likely to induce specific sports related FoM. As the PHOSA-ACLR items measure one latent trait, the items can be used in selecting items when treating fear of harm in sports related activities. Contrary to expectation, the average ACLR patients in this study did not score higher on FoM assessed using the PHOSA-ACLR when compared to individuals without ACLR. Time since 
ACLR reconstruction for patients in this study was on average 1 year and 3 months, at which time most patients are likely to have recovered to normal use. They did score higher in FoM compared to healthy junior professional soccer players, but lower compared to healthy first year physical therapy students.

The finding that ACLR patients on average scored lower on the PHOSA-ACLR than first year physical therapy students is intriguing. Several theoretical explanation come to mind. First of all, it is possible that implicit negative beliefs related to the actions depicted in the PHOSA-ACLR exist in healthy individuals as well, and first year PT students in particular, even without ever experiencing a negative effect of that action. This is consistent with the findings that people in the general population hold task specific dysfunctional beliefs which are inconsistent with current evidence[26]. For instance, task specific dysfunctional fear avoidance beliefs have been observed in healthy adults without back pain [27, 28], indicating that FoM might exist previous to an aversive event. As a result, people without pain display an implicit negative bias towards specific movements, such as bending and lifting, that are considered dangerous[29, 30]. Similar processes might also be at work in PT students when asked to judge the harmfulness of actions depicted in the PHOSA-ACLR photographs. These high scores of PT students are particular worrisome: health care providers fear avoidance beliefs and attitudes influence clinical practice[28, 31,32]. Furthermore, fear avoidance beliefs of physical therapist might influence fear beliefs in their patients[33]. Cultural differences offer an alternative explanation for the observed differences in fear-avoidance[34]. These differences in task specific FoM reported in this study might reflect differences in subcultures in which the participants are involved, resulting in differences in community beliefs communicated to the respondents[26]. ACLR athletes, aspiring professional athletes and amateur athletes have different social networks or subcultures. Such differences in social network, including spouses, friends, but also trainers, physical therapists etc. might account for differences in task specific FoM between groups. Finally, in patients with an ACL reconstruction exposure might be at work. Intensive exercises and training is needed to enhance the athlete's performance in sports activities[2]. Therefore, it is likely that patients are frequently exposed to the sports related activities assessed with the PHOSAACLR as part of their rehabilitation, diminishing levels of anxiety. The weak reversed relation between hours spend on sporting and PHOSA-ACLR score in the athletes without ACLR suggests that FoM for a specific situation diminishes when people are frequently exposed to that situation. More research is needed to determine how these interacting mechanisms are at work in athletes with and without ACLR.

This study is not without its limitations. First of all, patients with ACLR were recruited at different times after the ACLR, and this might have an impact on the average score reported in this sample. However, PHOSA-ACLR was assessed between 3-36 months after the intervention, at which time most patients have recovered to normal use. Furthermore, time since ACLR in this sample is unrelated to PHOSA-ACLR score [20]. The average scores on PHOSA-ACLR is lower compared to the average scores in the students, but higher in junior professional soccer players. A second limitation of the study is the sample of student starting with the study Physical Therapy. These students might have been primed on possible negative consequences of certain behaviours. However, to rule out such a bias as much as possible, the questionnaire was completed by the students at the start of the first college on an unrelated topic. As physical therapy student are known to be frequent sporters they were considered to be a good control 
group. However, to rule out any bias more research is needed including samples of healthy subject with no previous concerns about sports injuries. A final restriction of the study is the different ways the PHOSA-ACRL was assessed. In two sample this was done on paper, in one sample online. Although there is no indication that these differences in assessment resulted in different scores, this cannot be entirely ruled out.

Nevertheless, the study showed that task specific FoM assessed with the PHOSA-ACLR is a unidimensional trait. FoM is a psychological barrier to returning to sports, particularly with ACL injuries were reinjury is very commonly observed[35]. Therefore, clinical practitioners should be aware of and monitor such fear of reinjury or FoM in ACL patients. Identifying at-risk patients with high levels of FoM, encouraging a multidisciplinary approach to patient care, and providing early referral to a sports psychologist may improve patient outcomes and increase return-to-play rates among athletes(Christino et al., 2015). In patients with high levels of FoM, exposure cognitive behavioural therapy can be applied. Exposure involves disputing dysfunctional thoughts and images by systematically questioning the reality of the thought and images, starting with the least fear inducing situation in the hierarchy. Such engagement can be either real or imagined. Imagery exposure (motor imagery) can potentially reduce the fear of re-injury and pain in ACLR patients(Rodriguez, Marroquin, \& Cosby, 2019).

Further research is needed to determine at what moment in the rehabilitation process FoM should be assessed. FoM is likely to be high shortly after the intervention but will decrease over time. However, longitudinal research is needed to determine at what point in time individual patients develop dysfunctional FoM, that is, FoM that will prevent return to sport. It is important to determine the earliest point of time in rehabilitation when dysfunctional FoM can be determined. Initiating exposure based treatment at that point in time is likely to be most beneficial.

\section{Conclusion}

Specific sports related FoM assessed with the PHOSA-ACLR is a unidimensional trait. In clinical practice the PHOSA-ACLR can be used to assess levels of sports related FoM in patients with ACLR, and physical therapists can use the PHOSA-ACLR to guide exposure treatment.

\section{List Of Abbreviations}

ACL: Anterior Cruciate Ligament

ACLR: Anterior Cruciate Ligament Reconstruction

ADLA: ctivities of Daily Life

ANCOVA: Univariate Analysis of Covariance

FoM: Fear of Movement

Page $10 / 20$ 
GRM: Graded Response Model

IRT: Item Response Theory

PHODA: Photographic Series of Daily Activities

PHOSA-ACLR: Photographic Series of Sport Activities related to ACLR

KOOS: Knee injury and Osteoarthritis Outcome Scale

SD: Standard Deviation

TSK: Tampa Scale of Kinesophobia

QOL: Quality of Life

\section{Declarations}

Ethics approval and consent to participate.

The study was conducted in accordance with the WMA declaration of Helsinki. All participants received information about the study, both verbally and in writing, and gave their informed consent in writing. The study including patients was approved by the Ethical Advisory Board, Faculty of Health, Behavior and Society of the HAN University of Applied Sciences. Committee reference number is ACPO 21.03/16. The study including students was approved by the HAN university of Applied Sciences Faculty of Health, Behaviour and Society Ethical Advisory Board; EACO 71.05/17.

Availability of data and materials

The raw data in a de-identified form are available and could be provided from the first author on reasonable request.

Competing interests.

The author(s) declare that they have no competing interest.

Funding.

No funding was received for this work.

Author's contribution.

WvL conceived of the study, and participated in its design, performed the statistical analysis, and coordination and drafted the manuscript. NvM, and RvC included patients in the study, and did help to draft the manuscript. BS participated in the design of the study and helped draft the manuscript. All authors read and approved the final manuscript. 


\section{Acknowledgments}

This study was possible with the help of the following students: A. van Kesteren, J. Roos, M. van Klaveren, M. van Til, C. van der Sluijs, Y Peeters, P. Rhebergen, A. Wassink; Yvonne van Dam.

\section{References}

1. Sanders TL, Maradit Kremers H, Bryan AJ, Larson DR, Dahm DL, Levy BA, Stuart MJ, Krych AJ. Incidence of Anterior Cruciate Ligament Tears and Reconstruction: A 21-Year Population-Based Study. Am J Sports Med. 2016;44(6):1502-1507.

2. van Melick N, van Cingel RE, Brooijmans F, Neeter C, van Tienen T, Hullegie W, Nijhuis-van der Sanden MW. Evidence-based clinical practice update: practice guidelines for anterior cruciate ligament rehabilitation based on a systematic review and multidisciplinary consensus. Br J Sports Med. 2016;50(24):1506-1515.

3. Kvist J, Ek A, Sporrstedt K, Good L. Fear of re-injury: a hindrance for returning to sports after anterior cruciate ligament reconstruction. Knee surgery, sports traumatology, arthroscopy. 2005;13(5):393397.

4. Flanigan DC, Everhart JS, Pedroza A, Smith T, Kaeding CC. Fear of reinjury (kinesiophobia) and persistent knee symptoms are common factors for lack of return to sport after anterior cruciate ligament reconstruction. Arthroscopy: The Journal of Arthroscopic \& Related Surgery. 2013;29(8):1322-1329.

5. Hsu C, Meierbachtol A, George SZ, Chmielewski TL. Fear of reinjury in athletes: implications for rehabilitation. Sports health. 2017;9(2):162-167.

6. Czuppon S, Racette BA, Klein SE, Harris-Hayes M. Variables associated with return to sport following anterior cruciate ligament reconstruction: a systematic review. Br J Sports Med. 2014;48(5):356-364.

7. Heil J. The injured athlete. Emotions in sport. 2000:245-265.

8. Trigsted SM, Cook DB, Pickett KA, Cadmus-Bertram L, Dunn WR, Bell DR. Greater fear of reinjury is related to stiffened jump-landing biomechanics and muscle activation in women after ACL reconstruction. Knee Surgery, Sports Traumatology, Arthroscopy. 2018:1-8.

9. Vlaeyen JW, Linton SJ. Fear-avoidance model of chronic musculoskeletal pain: 12 years on. Pain. 2012;153(6):1144-1147.

10. Ardern CL, Taylor NF, Feller JA, Webster KE. A systematic review of the psychological factors associated with returning to sport following injury. Br J Sports Med. 2013;47(17):1120-1126.

11. Everhart JS, Best TM, Flanigan DC. Psychological predictors of anterior cruciate ligament reconstruction outcomes: a systematic review. Knee Surgery, Sports Traumatology, Arthroscopy. 2015;23(3):752-762.

12. Lundberg $M$, Larsson $M$, Ostlund $H$, Styf J. Kinesiophobia among patients with musculoskeletal pain in primary healthcare. J Rehabil Med. 2006;38(1):37-43. 
13. Gatchel RJ, Neblett R, Kishino N, Ray CT. Fear-avoidance beliefs and chronic pain. Journal of Orthopaedic \& Sports Physical Therapy. 2016;46(2):38-43.

14. Ardern CL, Kvist J. What is the evidence to support a psychological component to rehabilitation programs after anterior cruciate ligament reconstruction? Current Orthopaedic Practice. 2016;27(3):263-268.

15. Christino MA, Fantry AJ, Vopat BG. Psychological Aspects of Recovery Following Anterior Cruciate Ligament Reconstruction. J Am Acad Orthop Surg. 2015;23(8):501-509.

16. Vlaeyen J, Morley S, Linton SJ, Boersma K, de Jong J. Pain-related fear: exposure based treatment for chronic pain: IASP press; 2012.

17. McArdle S. Psychological rehabilitation from anterior cruciate ligament-medial collateral ligament reconstructive surgery: a case study. Sports health. 2010;2(1):73-77.

18. Miller, R.- Kori, S.- Todd, D. The tampa scale for kinisophobia. Unpublished report.. 1991.

19. Webster KE, Feller JA, Lambros C. Development and preliminary validation of a scale to measure the psychological impact of returning to sport following anterior cruciate ligament reconstruction surgery. Physical therapy in sport. 2008;9(1):9-15.

20. van Lankveld W, van Melick N, Habets B, Roelofsen E, Staal JB, van Cingel R. Measuring individual hierarchy of anxiety invoking sports related activities: development and validation of the Photographic Series of Sports Activities for Anterior Cruciate Ligament Reconstruction (PHOSAACLR). BMC musculoskeletal disorders. 2017;18(1):287.

21. Tesio L. Outcome measurement in behavioural sciences: a view on how to shift attention from means to individuals and why. International Journal of Rehabilitation Research. 2012;35(1):1-12.

22. Roos EM, Roos HP, Lohmander LS, Ekdahl C, Beynnon BD. Knee Injury and Osteoarthritis Outcome Score (KOOS)-development of a self-administered outcome measure. Journal of Orthopaedic \& Sports Physical Therapy. 1998;28(2):88-96.

23. Vlaeyen JW, Kole-Snijders AM, Boeren RG, Van Eek H. Fear of movement/(re) injury in chronic low back pain and its relation to behavioral performance. Pain. 1995;62(3):363-372.

24. Lord FM. Applications of item response theory to practical testing problems: Routledge; 2012.

25. Cohen J. A power primer. Psychol Bull. 1992;112(1):155.

26. Darlow B. Beliefs about back pain: the confluence of client, clinician and community. International Journal of Osteopathic Medicine. 2016;20:53-61.

27. Buer N, Linton SJ. Fear-avoidance beliefs and catastrophizing: occurrence and risk factor in back pain and ADL in the general population. Pain. 2002;99(3):485-491.

28. Houben RM, Ostelo RW, Vlaeyen JW, Wolters PM, Peters M, Stomp-van den Berg SGM. Health care providers' orientations towards common low back pain predict perceived harmfulness of physical activities and recommendations regarding return to normal activity. European Journal of Pain. 2005;9(2):173-183. 
29. Caneiro J, O'Sullivan P, Lipp OV, Mitchinson L, Oeveraas N, Bhalvani P, Abrugiato R, Thorkildsen S, Smith A. Evaluation of implicit associations between back posture and safety of bending and lifting in people without pain. Scandinavian journal of pain. 2018;18(4):719-728.

30. Goubert L, Crombez G, Hermans D, Vanderstraeten G. Implicit attitude towards pictures of backstressing activities in pain-free subjects and patients with low back pain: an affective priming study. European Journal of Pain. 2003;7(1):33-42.

31. Gardner T, Refshauge K, Smith L, McAuley J, Hübscher M, Goodall S. Physiotherapists' beliefs and attitudes influence clinical practice in chronic low back pain: a systematic review of quantitative and qualitative studies. Journal of physiotherapy. 2017;63(3):132-143.

32. Coudeyre E, Rannou F, Tubach F, Baron G, Coriat F, Brin S, Revel M, Poiraudeau S. General practitioners' fear-avoidance beliefs influence their management of patients with low back pain. Pain. 2006;124(3):330-337.

33. Lakke SE, Soer R, Krijnen WP, van der Schans, Cees P, Reneman MF, Geertzen JH. Influence of Physical Therapists' Kinesiophobic Beliefs on Lifting Capacity in Healthy Adults. Phys Ther. 2015;95(9):1224-1233.

34. Vlaeyen JW. The intricate relationship amongst pain intensity, fear and avoidance. Scandinavian journal of pain. 2016;13(1):128-129.

35. Ardern CL, Taylor NF, Feller JA, Webster KE. Fear of re-injury in people who have returned to sport following anterior cruciate ligament reconstruction surgery. Journal of Science and Medicine in Sport. 2012;15(6):488-495.

36. Rodriguez RM, Marroquin A, Cosby N. Reducing fear of reinjury and pain perception in athletes with first-time anterior cruciate ligament reconstructions by implementing imagery training. J Sport Rehab. 2019;28(4):385-389.

\section{Tables}


Table 1: Characteristics of patients in rehabilitation after reconstruction surgery $(N=58)$

\begin{tabular}{llll|} 
& $\begin{array}{l}\text { Theoretical } \\
\text { Range }\end{array}$ & $\begin{array}{l}\text { Mean (Standard } \\
\text { Deviation) }\end{array}$ & $\begin{array}{l}\text { Observed } \\
\text { Range }\end{array}$ \\
KOOS pain & $0-100$ & $81.8(17.7)$ & $33-100$ \\
\hline KOOS symptoms & $0-100$ & $63.2(12.8)$ & $29-100$ \\
\hline KOOS Activities of Daily Life & $0-100$ & $89.1(14.2)$ & $35-100$ \\
\hline KOOS Sports and Leisure & $0-100$ & $59.1(30.6)$ & $00-100$ \\
KOOS Quality of Life & $0-100$ & $48.3(14.1)$ & $06-75$ \\
$\begin{array}{l}\text { Tampa Scale of } \\
\text { Kinesiophobia }\end{array}$ & $0-68$ & $35.7(7.1)$ & $20-51$ \\
Abbreviations: $\mathrm{N}=$ number of participants, KOOS = Knee Injury and Osteoarthritis Outcome Score \\
\hline
\end{tabular}

\begin{tabular}{|lll|}
\hline \multicolumn{2}{|l|}{ Table 2: Description of student samples (N = 199) } \\
& $\begin{array}{l}\text { Regular students } \\
\mathrm{N}=169\end{array}$ & Vitesse \\
& $\mathrm{N}=30$ \\
\hline Average age (95\% Cl) & $19.2(2.0)$ & $18.3(3.2)$ \\
\hline Average hours active sporting (95\% Cl) & $5.6(2.8)$ & $14.9(4.2)$ \\
\hline Gender (\% male) & $48 \%$ & $94 \%$ \\
\hline Earlier complaints (\% yes) & $71 \%$ & $60 \%$ \\
\hline Knowledge of ACLR (\% yes) & $35 \%$ & $65 \%$ \\
\hline Chronic condition (\% yes) & $10 \%$ & $7 \%$ \\
\hline High risk sports (\% yes) & $66 \%$ & $100 \%$ \\
\hline
\end{tabular}


Table 3: Phosa items, observed range, means, standard deviations, and median score.

\begin{tabular}{|llll|}
\hline Sports related activity & Observed Range & Mean score (SD) & Median \\
\hline 1. Running & $0-10$ & $3.67(2.73)$ & 3.0 \\
\hline 2. Landing after jumping & $0-10$ & $4.39(2.90)$ & 4.0 \\
\hline 3. Squats & $0-10$ & $3.11(2.63)$ & 3.0 \\
\hline 4. Lateral lunging & $0-10$ & $4.76(2.83)$ & 5.0 \\
\hline 5. Single leg jump & $0-10$ & $3.88(2.71)$ & 4.0 \\
\hline 6. Sliding & $0-10$ & $3.98(2.79)$ & 4.0 \\
\hline 7. Bring to a halt & $0-10$ & $5.11(2.85)$ & 6.0 \\
\hline 8. Hop & $0-10$ & $4.11(2.84)$ & 4.0 \\
\hline 9. Lunge & $0-10$ & $3.77(2.78)$ & 4.0 \\
\hline 10. Start to sprint & $0-10$ & $4.09(2.84)$ & 4.0 \\
\hline 11. Jumping on a trampoline & $0-10$ & $4.06(2.77)$ & 4.0 \\
\hline 12. Pivoting movement & $0-10$ & $5.68(2.86)$ & 6.0 \\
\hline
\end{tabular}




\begin{tabular}{|l|r|r|r|r|r|r|r|r|r|r|r|}
\hline Table 4: location parameters of the PHOSA items, sorted from lowest difficulty to highest. \\
\hline & $\beta .1$ & $\beta .2$ & $\beta .3$ & $\beta .4$ & $\beta .5$ & $\beta .6$ & $\beta .7$ & $\beta .8$ & $\beta .9$ & $\beta .10$ & $\beta$ \\
& & & & & & & & & & & \\
\hline 6. Sliding & -2.73 & -2.03 & -1.10 & -0.20 & 0.43 & 1.15 & 1.80 & 2.64 & 3.50 & 4.73 & 1.69 \\
\hline 3. Squats & -2.42 & -1.15 & -0.19 & 0.80 & 1.59 & 2.30 & 2.70 & 3.75 & 5.82 & 7.25 & 2.18 \\
\hline 1. Running & -3.30 & -2.04 & -0.63 & 0.30 & 0.86 & 1.79 & 2.73 & 3.85 & 5.39 & 5.96 & 2.40 \\
\hline 11. Jumping & -4.31 & -2.56 & -1.32 & -0.35 & 0.49 & 1.24 & 1.92 & 3.52 & 4.42 & 6.19 & 2.42 \\
\hline 9. Lunge & -3.40 & -2.03 & -0.97 & -0.07 & 0.70 & 1.63 & 2.53 & 3.47 & 5.34 & 7.82 & 2.47 \\
\hline 4. Lateral Lunging & -4.48 & -3.45 & -2.49 & -1.60 & -0.40 & 0.65 & 1.36 & 2.76 & 4.12 & 5.67 & 2.49 \\
\hline 5. Leg jump & -3.95 & -2.33 & -1.33 & -0.28 & 0.61 & 1.72 & 2.58 & 3.89 & 4.73 & 5.76 & 2.56 \\
\hline 12. Pivoting & -5.92 & -5.32 & -3.56 & -2.59 & -1.91 & -1.03 & 0.37 & 1.44 & 2.80 & 4.03 & 2.58 \\
\hline 7. Bring to Halt & -5.08 & -3.84 & -3.01 & -1.79 & -0.98 & -0.08 & 1.00 & 2.14 & 3.85 & 5.84 & 2.59 \\
\hline 2. Landing & -4.73 & -3.12 & -1.74 & -0.69 & 0.31 & 1.02 & 1.67 & 3.14 & 5.23 & 7.28 & 2.92 \\
\hline 10. Start sprint & -4.46 & -3.28 & -1.81 & -0.40 & 0.63 & 1.49 & 2.77 & 3.92 & 5.88 & 8.81 & 3.39 \\
\hline 8. Hop & -4.69 & -3.09 & -2.11 & -0.53 & 0.68 & 1.43 & 2.78 & 4.12 & 6.07 & 7.67 & 3.48 \\
\hline
\end{tabular}

B.1 = Beta.1 represents the threshold between response category 0 and 1, Beta.2 represents the threshold between response category 1 and 2, etc. The final Beta indicates the item's overall difficulty; the ability (theta) associated with the weighted threshold between response category 0 and 10 . 
Table 6: Average PHOSA score, range, standard deviation (S.D.) and 95\% Confidence Interval for patients after ACLR, regular students, and soccer players.

\begin{tabular}{|lllll|} 
& ACLR & Regular students & Soccer & Total \\
& & $\mathrm{N}=169$ & $\mathrm{~N}=30$ & $\mathrm{~N}=257$ \\
\hline Average PHOSA & 3.1 & 4.9 & 2.6 & 4.2 \\
\hline Range & $0-10$ & $0-10$ & $0-10$ & $0-10$ \\
\hline Standard Deviation (SD) & 2.3 & 2.0 & 2.7 & 2.3 \\
$95 \% \mathrm{Cl}$ & $2.5-3.7$ & $4.6-5.2$ & $1.6-3.6$ & $3.9-4.5$ \\
\hline Median & 2.5 & 5.5 & 1.6 & 4.8 \\
\hline
\end{tabular}

\section{Figures}

\section{Item Information Curves}

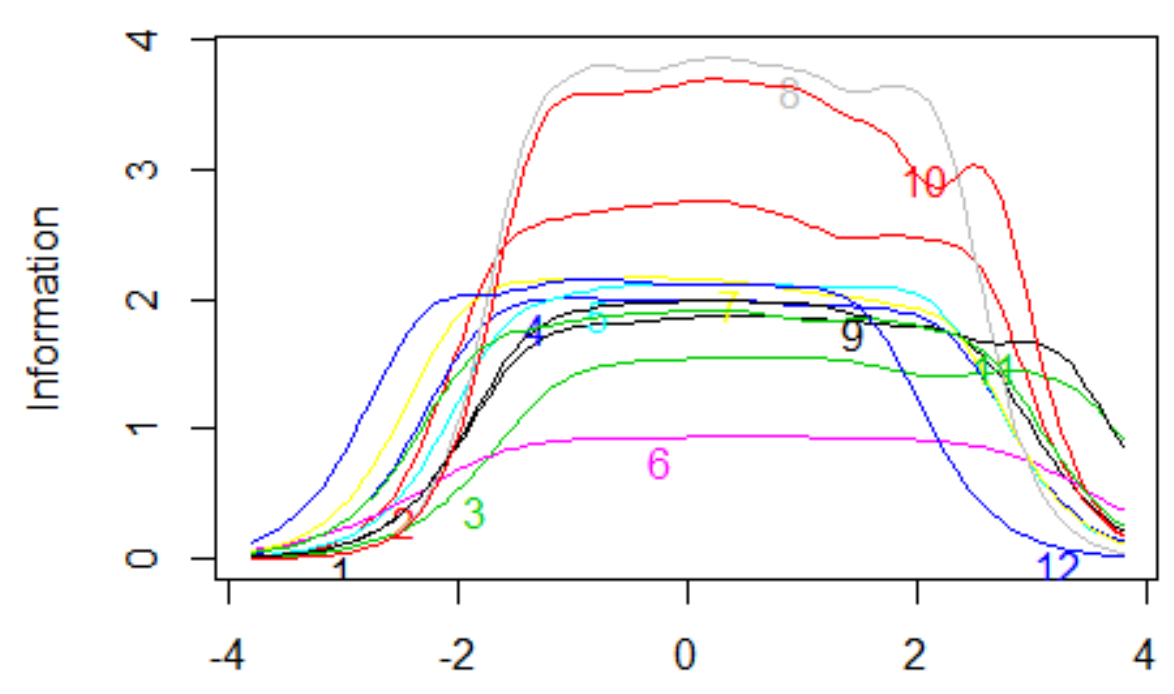

Ability

\section{Figure 1}

Item Information Curves (IIC) for the 12-item PHOSA. The amount of item-information (y-axis) is plotted against latent ability scores ( $\mathrm{x}$-axis). Each colored line represents an item, and how much information a response on that item provides at increasing standardized levels of ability (theta). For example, item 6 
carries relatively low information across all ability levels, whereas answers items 8 and 10 are highly informative in the -2 to +3 ability range.

\section{Kernel Density Estimation for Ability Estimates}

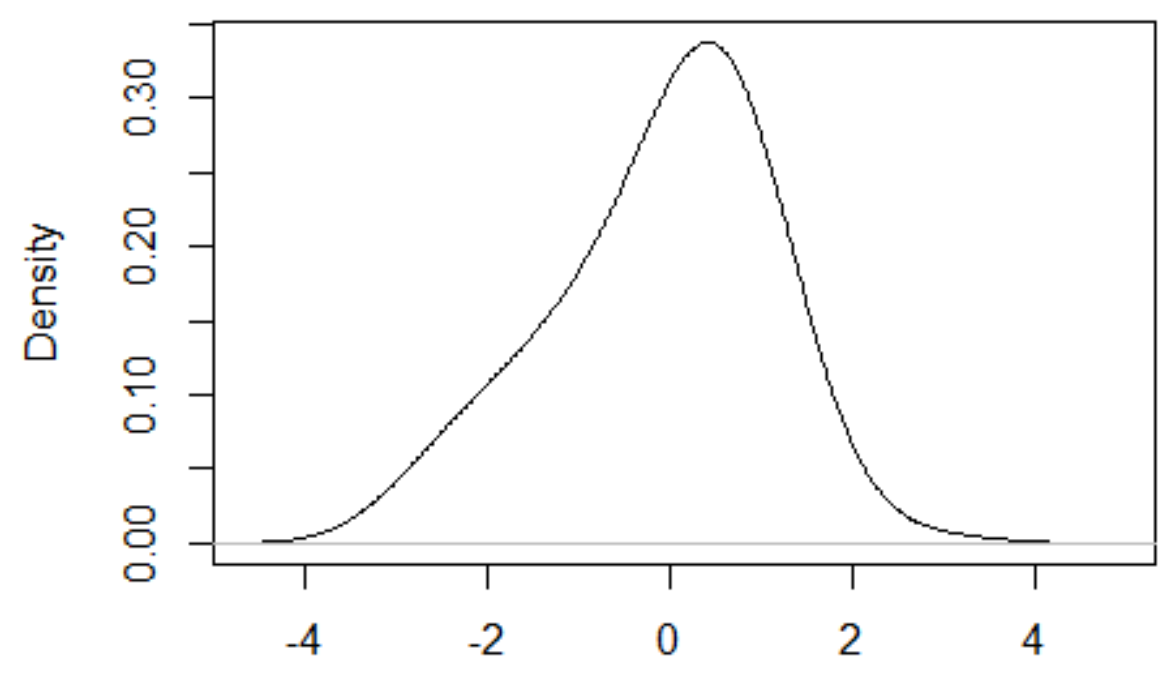

Ability

Figure 2

Density plot of the 12-item PHOSA visualizing the distribution of latent PHOSA scores (x-axis).

\section{Test Information Function}

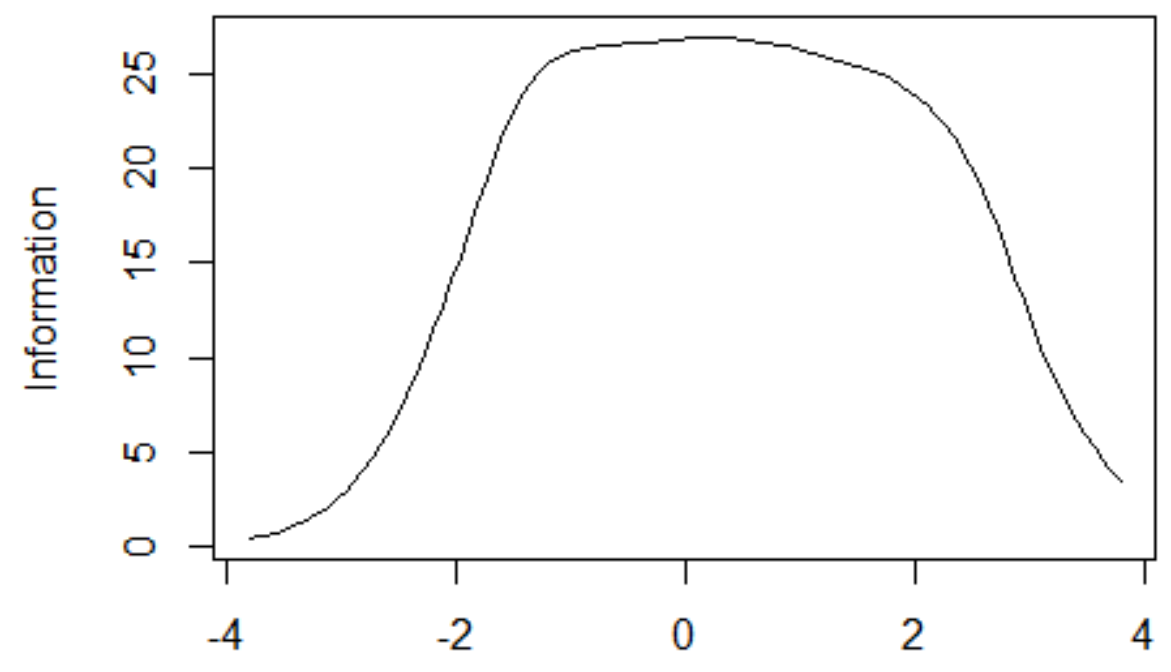

Ability

Figure 3 
A visualization of the Test Information Function for the 12-item PHOSA over the range of ability scores (xaxis). 